\title{
Recruitment of Spinal Motor Pools during Voluntary Movements versus Stepping after Human Spinal Cord Injury
}

\author{
M. MAEGELE, ${ }^{1}$ S. MÜLLER, ${ }^{2}$ A. WERNIG, ${ }^{1}$ V.R. EDGERTON, ${ }^{3,4,5}$ and S.J. HARKEMA ${ }^{3,6}$
}

\begin{abstract}
We investigated the activation of lower limb motor pools by supraspinal and spinal networks after human spinal cord injury (SCI). We compared electromyographic (EMG) activity from six muscles during voluntarily attempted non-weight-bearing single-joint movements, multijoint movements approximating stepping in a supine position, and weight-bearing stepping on a treadmill with body weight support (BWST) in seven clinically incomplete and three clinically complete SCI subjects. Seven SCI subjects had previously completed Laufband therapy (a specific step training using variable levels of body weight support and manual assistance). Significant coactivation of agonists and antagonists and multijoint flexion or extension movements of the entire limb occurred during attempts at isolated knee or ankle single-joint movements in clinically incomplete SCI subjects. Further, some muscles that were not recruited during voluntary attempts at single-joint movements were activated during voluntary step-like multijoint movements (5/16 comparisons). This suggests that the residual voluntary motor control in incomplete SCI subjects evokes more generalized motor patterns (limb flexion or extension) rather than selective activation of individual muscles. Clinically incomplete and clinically complete SCI subjects could achieve greater activation of motor pools and more reciprocal patterns of activity between agonists and antagonists during weight bearing stepping than during non-weight-bearing voluntary movements. The EMG mean amplitudes were higher during stepping than during voluntary movements in 50/60 muscles studied $(p<0.05)$. These results suggest that stepping with knee and hip extension and flexion and alternating lower limb loading and unloading provides proprioceptive inputs to the spinal cord that increases motor recruitment and improves reciprocity between agonists and antagonists compared to voluntary efforts.
\end{abstract}

Key words: coactivation; Laufband therapy; locomotion; locomotor training; motor recruitment; proprioception; spinal cord injury

\section{INTRODUCTION}

$\mathbf{T}$ he Selection and Level of activation of the lower limb motor neurons can be modulated effectively by descending supraspinal input to the lumbosacral spinal cord in humans. After spinal cord injury (SCI), the functionality of descending control of motor pools is estimated from movements that the individual can volitionally generate in selected muscle groups. This motor output is also routinely used as a predictor of the level

${ }^{1}$ Department of Physiology, University of Bonn, Bonn, Germany.

${ }^{2}$ Klinikum Karlsbad-Langensteinbach, Karlsbad, Germany.

The ${ }^{3}$ Brain Research Institute, ${ }^{4}$ Department of Physiological Sciences, ${ }^{5}$ Department of Neurobiology, and ${ }^{6}$ Department of Neurology, University of California Los Angeles, Los Angeles, California. 
of ambulation that will be regained after neurological injury (Crozier et al., 1992; Hussey and Stauffer, 1973; Waters et al., 1989, 1993, 1994). The assumption implied by this assessment is that the limitation in the level of voluntary control of individual muscle groups of the lower limbs is the only factor that predicts locomotor capacity. Acceptance of this assumption then presumes that the sensory input associated with load-bearing stepping is of limited functional relevance in the ability to execute standing and stepping.

However, sensory input plays an essential role in regulating both the level of activation and the coordination of motor pools involved in stepping as demonstrated by a wide range of studies in laboratory animals (Andersson and Grillner, 1981, 1983; Barbeau et al., 1993; Barbeau and Rossignol, 1987; Conway et al., 1987; de Leon et al., 1998a; Duysens and Pearson, 1980; Gossard and Hultborn, 1991; Grillner and Rossignol, 1978; Grillner and Zangger, 1979; Lovely et al., 1986; Pearson and Duysens, 1976). Although sensory input derived from the muscles, tendons, joints and skin of the lower limbs of humans can influence the mechanics of load-bearing stepping, it is not known whether this influence is dependent on simultaneously occurring voluntary control from supraspinal centers (Aniss et al., 1992; Burke et al., 1991; Capaday and Stein, 1986; Crenna and Frigo, 1987; Duysens et al., 1990; Yang and Stein, 1990). Evidence from severely impaired SCI subjects suggests that the level of activation of motor pools involved in stepping using body weight support on a treadmill (BWST) can be influenced significantly by peripheral sensory input (Barbeau 1987; Barbeau and Blunt, 1991; Dietz et al., 1994, 1995, 1998a,b; Dobkin et al., 1995; Harkema et al., 1997; Stewart et al., 1991; Wernig and Müller, 1991, 1992; Wernig et al., 1995, 1999, 2000).

We hypothesized that the level of activation of motor pools during load-bearing stepping exceeds the level that a severely injured individual following SCI can develop voluntarily without limb loading. We compared the EMG from six lower limb muscles of SCI subjects during voluntarily attempted non-weight-bearing single-joint movements, multijoint movements approximating stepping in a supine position, and weight-bearing stepping using BWST. Clinically incomplete and clinically complete SCI subjects demonstrated a greater level of motor neuron activation and less coactivation among antagonistic muscles during load-bearing stepping than during voluntary attempted movements. We also found that multiple muscle groups were activated (flexion and extension and even multiple joints) when single-joint flexion or extension movements were intended in subjects with reduced descending activity following SCI. These data demonstrate that step-associated proprioception can co- ordinate spinal motor pools in an effective temporal sequence and at an adequate level of activation for generating stepping even when an individual is not able to voluntarily execute similar motor patterns in defined resting positions.

\section{MATERIALS AND METHODS}

\section{Subject Population}

Ten SCI subjects (eight male, two female; 13-61 years; $29-87 \mathrm{~kg}$ ) volunteered for this study. Clinical characteristics of all subjects are summarized in Table 1. A clinician classified the subjects with the use of the American Spinal Injury Association (ASIA) impairment scale, which categorizes individuals by their sensorimotor function below the level of lesion and designates a cumulative motor score (Ditunno et al., 1994; Maynard et al., 1997). In brief, the ASIA impairment scale classifies individuals as follows: $\mathrm{A}=$ Complete-no motor or sensory function is preserved below the lesion including the sacral segments S4-S5; B = incomplete-sensory but not motor function is preserved below the neurological level and includes the sacral segments S4-S5; C = incomplete-motor function is preserved below the neurological level, and more than half of the key muscles below the neurological level have a muscle grade less than 3; $\mathrm{D}=$ incomplete-motor function is preserved below the neurological level, and at least half of the key muscles below the neurological level have a muscle grade of 3 or more; or $\mathrm{E}=$ normal-motor and sensory function is normal. In one subject (A10), transcranial magnetic stimulation of the motor cortex and sensory evoked potentials (recorded from the popliteal fossa, lumbar spine, and scalp during tibial nerve stimulation) both reported absence of detectable supraspinal conductivity to the lower limbs.

Motor function and ambulatory status before and after Laufband therapy is summarized in Table 2. Some characteristics from these subjects presented here have previously been reported (Wernig et al., 1995, 1999). A recently established functional locomotor assessment (EU Walking) was performed (Wernig et al., 1995, 1999). In brief, walking capability was tested by assessment of walking with and without assistive devices by three independent observers. EU Walking defines functional classes: 0 = cannot walk even with moderate help of two therapists, 1 = can walk with moderate help of two therapists, $2=$ can walk at the railing with active help by one therapist, $3=$ can walk without help by other persons using a rollator/walker, $4=$ can walk independently using two canes, $5=$ can walk without devices. Classes 0-2 indicate dependence on help by others, and 3-5 indepen- 
Table 1. Clinical Characteristics of Subjects Studied

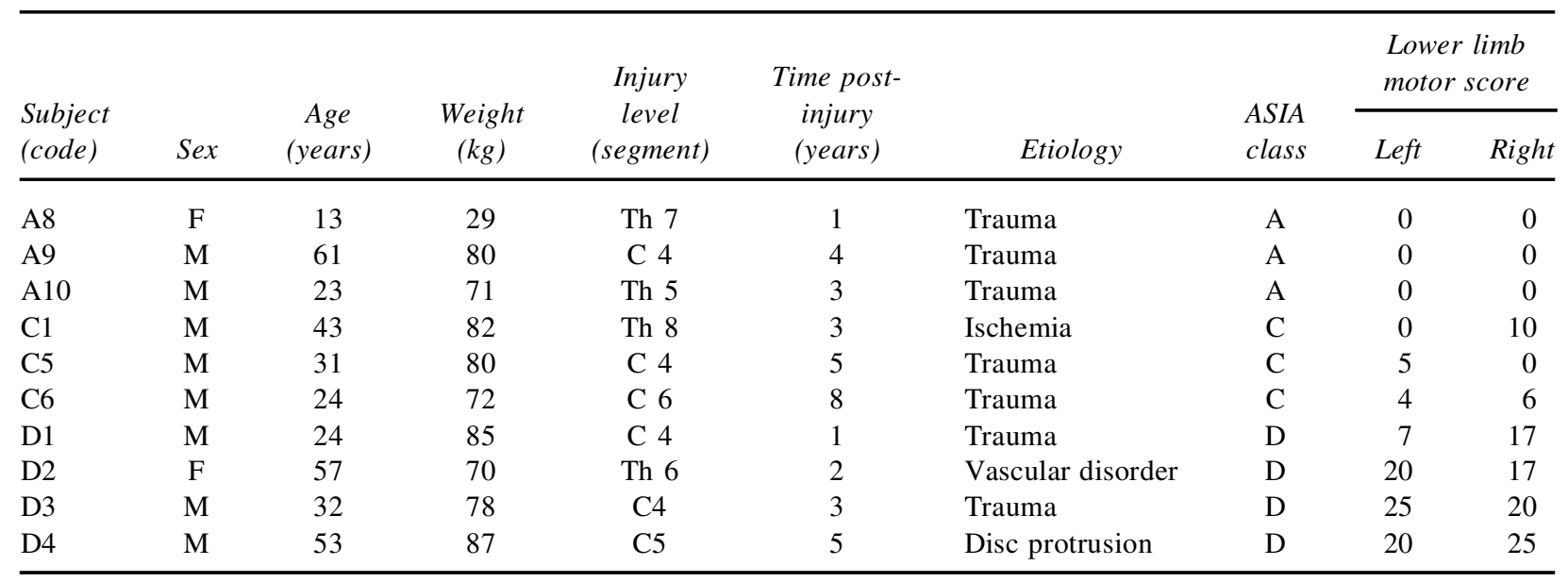

Th, thoracic; $\mathrm{C}$, cervical.

dence for locomotion as defined. Reliability (reliability index/interrater consistency $/ K=0.84$ ) was determined by comparing the independent assessments of 61 film sequences by two investigators (Cohen, 1960). The inability to use walking devices in quadriplegics due to severe arm paralyses was assessed separately, since it may limit the rating of such patients to classes 1 to 2 regardless of lower limb function. In this study, some subjects had moderate (C5 and D3) or severe (D1 and D4) difficulties in holding assistive devices such as canes or walkers due to upper extremity paralysis (Table 2). Also, the Cumulative Muscle Index (CMI) scored voluntary activity in defined resting positions on a scale from 0 to 5 (with half values in between; Kendall et al., 1993) for the gluteals (maximus and medius/minimus), iliopsoas, sartorius, quadriceps, ischiocrurales, triceps surae and tibialis anterior. This index differs from the ASIA classification in the specific muscles tested and is not limited to key muscles for a given segment. The CMI is restricted to the lower limb muscles so that in quadriplegics CMI values may be high while EU Walking scores may not exceed 1 (individuals need moderate assistance from two people to walk) because of arm weakness.

Experiments were performed at the Klinikum Karlsbad-Langensteinbach, Germany (subjects A8-A10; C5C6; D1-D4) and at the University of California Los Angeles (subject C1). The Klinikum Karlsbad-Langensteinbach and the University of California at Los Angeles Human Subjects Protection Committees approved all experiments and all procedures were within the guidelines of the code of ethics of the World Medical Association. Each subject signed an informed consent form prior to the experiments.

\section{Experimental Design}

We measured EMG activity and joint kinematics during volitionally attempted single- and multijoint movements without limb loading and during stepping with

Table 2. EU Walking and the Cumulated Muscle Index for Subjects that Completed Laufband Therapy

\begin{tabular}{lcccccc}
\hline \multirow{2}{*}{$\begin{array}{l}\text { Subject } \\
\text { (code) }\end{array}$} & \multirow{2}{*}{$\begin{array}{c}\text { EU } \\
\text { (pretraining) }\end{array}$} & $\begin{array}{c}\text { EU } \\
\text { (posttraining) }\end{array}$ & Before & After & Before & CMI, right \\
\hline A8 & 0 & 0 & 0 & 0 & 0 & 0 \\
A10 & 0 & 0 & 0 & 0 & 0 & 0 \\
C5 & 0 & $0^{*}$ & 7.5 & 8.5 & 0 & 0 \\
D1 & 1 & 4 & 9 & 9.5 & 15.5 & 16 \\
D2 & 1 & $4^{* *}$ & 24 & 26 & 9.5 & 10.5 \\
D3 & 2 & $3^{* *}$ & 10 & 27 & 11 & 10.5 \\
D4 & 2 & & 10.5 & 14 & 14 \\
\hline
\end{tabular}

The *moderate or $* *$ severe difficulties in holding assistive devices are due to upper extremity paralysis and have a restrictive effect on independentambulation. 
manual assistance using BWST. During volitionally attempted single-joint movements at rest without limb loading, the subjects were lying on their side to neutralize gravitational effects on extensor-flexor movements. The trainer held the leg in a neutral abduction-adduction position and slightly flexed the hip and knee to avoid extensor spasms. In this position, we requested subjects to flex or extend the ankle or the knee joint (dorsi-/ plantarflexion and knee flexion/extension). Three consecutive trials were performed and after each trial the trainer returned the leg to the initial resting position. The subjects also volitionally attempted multijoint movements at rest without limb loading in a supine position with their hip and knee joints slightly flexed to avoid extensor spasms. We requested subjects to perform alternating flexion/extension movements of the legs that approximated stepping movements.

When stepping, a harness (Wernig, 2000) attached to suspension cables supported the subjects over a treadmill. Elastic cords at the subjects' hips or a trainer stabilized the pelvis. Trainers held each leg distal to the patella to assist with knee extension during the stance phase and at the popliteal fossa to assist knee flexion during the swing phase. Trainers also held each leg proximal to the ankle to assist with foot lift-off and placement when necessary (Table 1; A8, A9, A10, C1, C5, C6, D1, D4). Two subjects (Table 1, D2, D3) stepped independently on the treadmill. Four subjects (Tables 1 and 2; D1-D4) ambulated overground with different levels of assistance. We also recorded vertical ground reaction forces during stepping. The amount of body weight support (BWS) provided ranged from $15 \%$ to $56 \%$ and treadmill speeds ranged from 0.22 to $0.33 \mathrm{~m} / \mathrm{sec}$.

Four subjects (A8, A10, C5, D1) had previously received an average of 12 weeks of Laufband therapy (Wernig and Müller, 1991, 1992) using BWST and manual assistance ( $30 \mathrm{~min} /$ day; 5 days/week) at treadmill speeds of $0.05-0.42 \mathrm{~m} / \mathrm{sec}$. Characteristics of the motor performance are reported in Table 2 (also reported in part in Wernig et al., 1995, 1999, 2000). Three subjects (Table 2; D2, D3, D4) had just completed 12 weeks of Laufband therapy prior to the experiments. All training occurred at the Klinikum Karlsbad-Langensteinbach, Germany.

\section{Data Acquisition}

We collected EMG and joint angles at $1,000 \mathrm{~Hz}$ using a 24-channel hard-wired analog to digital board and a custom written LabView software acquisition program (National Instruments, Austin, TX). EMG data were AC coupled into a differential amplifier (Konigsberg Instruments, Pasedena, CA). The signals were pulse-interval modulated to a fiberoptic transmitter and relayed to de-
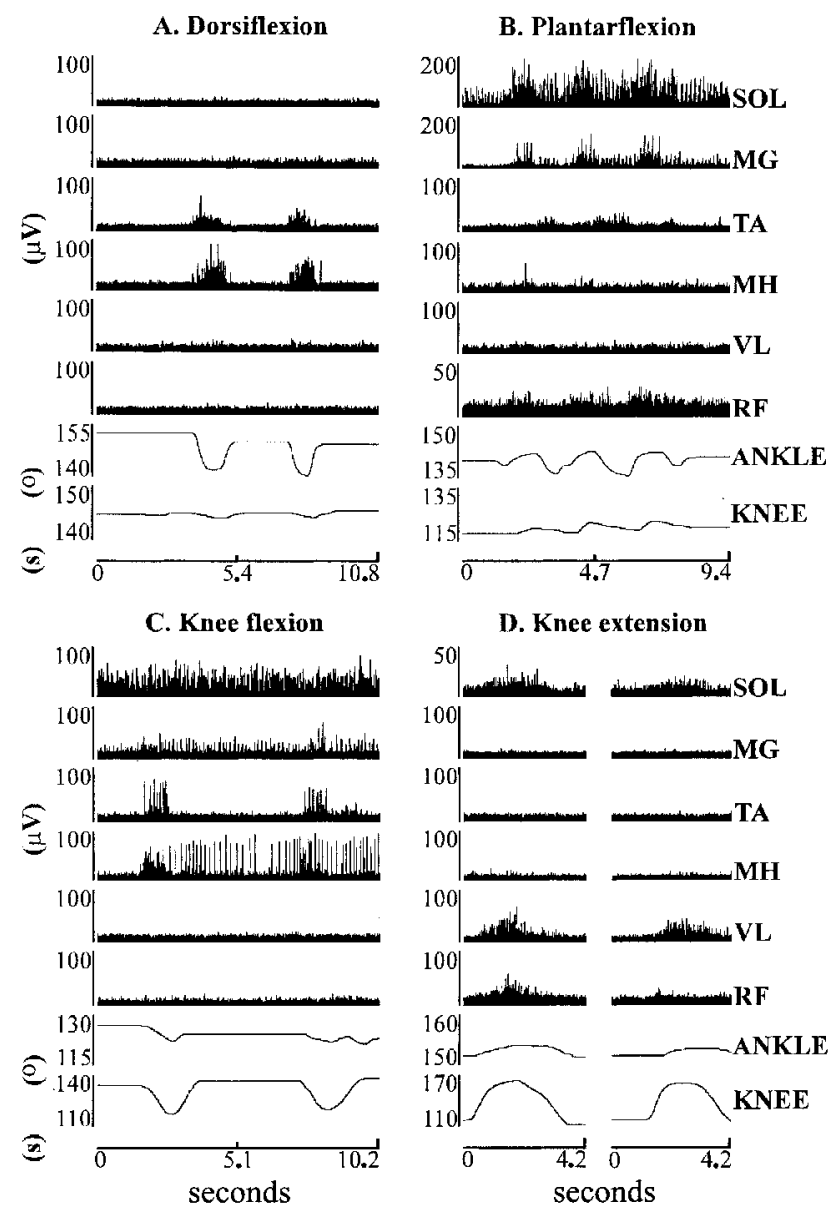

D. Knee extension

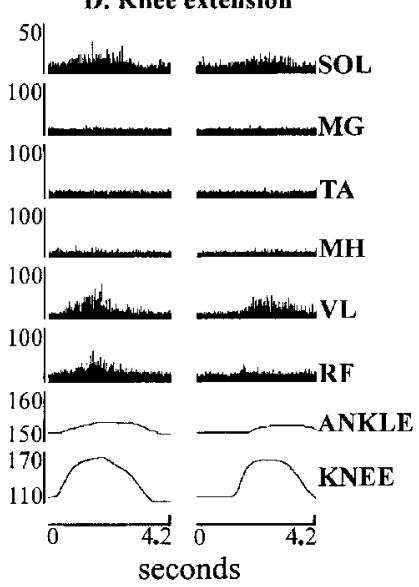

FIG. 1. Coactivation of functional agonists occurred during attempts at single-joint movements. Electromyographic (EMG) activity $(\mu \mathrm{V})$ from the soleus (SOL), medial gastrocnemius (MG), tibialis anterior (TA), medial hamstrings (MH), vastus lateralis (VL), and rectus femoris (RF); and knee and ankle angles $\left(^{\circ}\right)$ when clinically incomplete SCI subjects (C5, panel A; $\mathrm{C} 1$, panels B and C and D4, panel D; as referenced in Table 1) attempted to move a single-joint. The movement action requested by the experimenter was dorsiflexion(A), plantarflexion (B), knee flexion (C), and knee extension (D). Muscles showing EMG activity usually differed from the ones expected for the movement action requested by the experimenter. Both the knee and ankle joints moved in each case even though the subjects were asked to move only a single joint.

coding electronics. We measured EMG activity bilaterally from the soleus (SOL), medial gastrocnemius (MG), tibialis anterior (TA), medial hamstrings $(\mathrm{MH})$, vastus lateralis (VL), and rectus femoris (RF) muscles using bipolar surface electrodes (Harkema et al., 1997). We measured knee and ankle angles bilaterally with electrogoniometers placed over the axis of the joint rotation. Ankle angles were measured with the fibular head and 5th metatarsal as landmarks. We measured limb load by 
recording pressure distributon of the foot's plantar surface using pressure sensor shoe inserts (Tekscan, Boston, MA). We dynamically calibrated (Carollo et al., 1993) the Tekscan shoe inserts with a force platform (Kistler, Amherst, NY) using nondisabled subjects of comparable weight and shoe size to the SCI subjects (for details, see Harkema et al., 1997). Force signals from pressure sensors were interpolated from 50 to $1,000 \mathrm{~Hz}$ and synchronized with EMG and joint angle signals. In addition, we detected the stance and swing phases of the stepcycle using footswitches.

\section{Data Analyses}

We processed data using customized LabView software programs (National Instruments) by filtering EMG signals using a 4th order bandpass Butterworth filter $(40-500 \mathrm{~Hz})$ and rectification. As a measure of EMG amplitude, we calculated EMG mean amplitude as the sum of the amplitudes of the rectified EMG from burst onset to burst offset divided by burst duration. The onset of EMG bursts was defined as the time when the signal amplitude remained above the threshold (three standard deviations above baseline for $50 \mathrm{msec}$ and the offset when the signal amplitude remained below the threshold for $150 \mathrm{msec}$ ). We compared the EMG mean amplitude dur- ing volitionally attempted single-joint movements, multijoint movements, and stepping from one limb of each subject. In subjects with detectable motor function in both limbs determined by lower limb motor score testing (C6, D1-D4), the limb with the lowest motor score was selected for analyses because in some subjects the contralateral limb was essentially normal. In subjects with detectable motor activity in only one limb, that limb was selected (C1 and $\mathrm{C5}$ ) rather than making comparisons to no activity. In subjects with no detectable voluntary lower limb muscle activity in either limb (A8-A10), the left limb was arbitrarily selected for analysis.

A one-way analysis of variance was used to test for significant differences among the averages of the EMG mean amplitudes during single-joint movements, multijoint movements, and stepping with BWST. Pair-wise comparisons were done using $t$ tests with a Bonferroni correction for multiple comparisons.

\section{RESULTS}

\section{EMG Activity during Single-Joint Movements}

Clinically incomplete SCI subjects could rarely generate isolated activity in single extensor and/or flexor
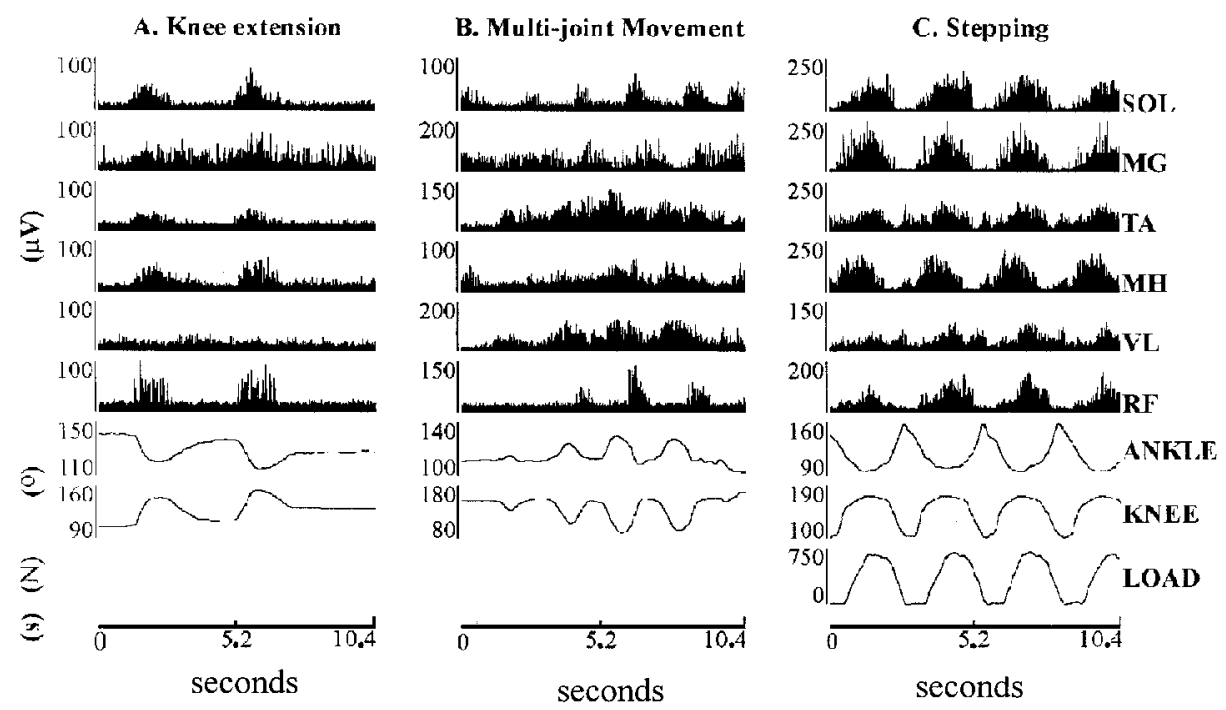

FIG. 2. Single- and multijoint movements and stepping from a clinically incomplete SCI subject (D3, reference Table 1) are shown. Electromyographic (EMG) activity $(\mu \mathrm{V})$ from the soleus (SOL), medial gastrocnemius (MG), tibialis anterior (TA), medial hamstrings $(\mathrm{MH})$, vastus lateralis (VL), and rectus femoris (RF); knee and ankle angles $\left(^{\circ}\right)$; and limb load in Newtons $(\mathrm{N})$ during an attempted single-joint movement $(\mathbf{A})$, multijoint movements $(\mathbf{B})$, and during weight-bearing stepping at $0.28 \mathrm{~m} / \mathrm{sec}$ with $15 \%$ body weight support (BWS) (C). The SOL, MG, MH, RF, and TA were activated, but not the VL, when knee extension was attempted, resulting in ankle dorsiflexion and knee extension (A). VL EMG activity was evident during multijoint movements (B) and stepping $(\mathbf{C})$. Coactivity of the VL and MH was greater in multijoint movements (B) than during stepping $(\mathbf{C})$. Alternating patterns of the SOL, and RF were observed during multijoint movements and stepping, however, only during stepping in the MG, TA, MH, and VL. 
muscles during attempted single-joint movements. Only $2 / 28$ attempts to perform single-joint movements while lying on a side (isolated dorsiflexion, plantarflexion, knee flexion, or knee extension; seven incomplete SCI subjects) resulted in the appropriate joint movement and EMG activity. In 6/28 attempted single-joint movements functional agonists of other joints of the same limb were activated generating flexion or extension patterns of the entire limb (Fig. 1). For example, during attempted dorsiflexion of the ankle, EMG activity was observed in the TA and also in the $\mathrm{MH}$ resulting in both ankle dorsiflexion and some knee flexion (Fig. 1A). Coactivation at the ankle and knee muscles was also observed in clinically incomplete SCI subjects during attempted plantarflexion resulting in both plantarflexion and knee extension (Fig. 1B). During attempted knee flexion, the $\mathrm{MH}$ and the TA were activated synchronously, and both the knee and the ankle were flexed (Fig. 1C). Similarly, both knee extension and plantarflexion occurred during attempts to only extend the knee (Fig. 1D).

In $12 / 28$ conditions, coactivation occurred in agonists and antagonists associated with either flexion or extension movements during attempts at single-joint movements. During both plantarflexion or knee extension efforts, EMG activity occurred in both knee extensors and plantarflexors as well as in the antagonistic TA and $\mathrm{MH}$ (Fig. 2A). Similarly, during attempted knee flexion or ankle dorsiflexion several muscles were activated. In one subject (D4), the TA was not activated during attempted dorsiflexion (Fig. 3A) but was evident when knee flexion was attempted (Fig. 3B). In 8/28 conditions minimal to no EMG activity was detected during voluntary attempts to move a single-joint (Figs. 3A and 4A). Clinically complete SCI subjects never generated EMG activity or joint movements during attempted single-joint movements.

\section{EMG Activity during Multi-Joint Movements and Load-Bearing Stepping}

Among the motor tasks attempted the clearest alternating burst patterns were observed during weightbearing stepping with independent alternating flexion and extension movements observed (Figs. 2-5). Individual muscles that were inactive during attempts to flex or extend a single-joint became active in 5/16 muscles during non-weight-bearing multijoint movements and in 15/16 muscles during weight-bearing stepping of incomplete SCI subjects. In a number of subjects, burst patterns were present during stepping, but not during single-joint or multijoint attempted movements (Figs. 3-5). Reciprocity of flexor and extensor bursts was more routinely observed during stepping than during multijoint movements, when the subjects were asked to approximate the kinematics of stepping. For example, the VL was coactivated with the MH during multijoint movement attempts (Fig. 2B) but was coactivated less during stepping (Fig. 2C). When
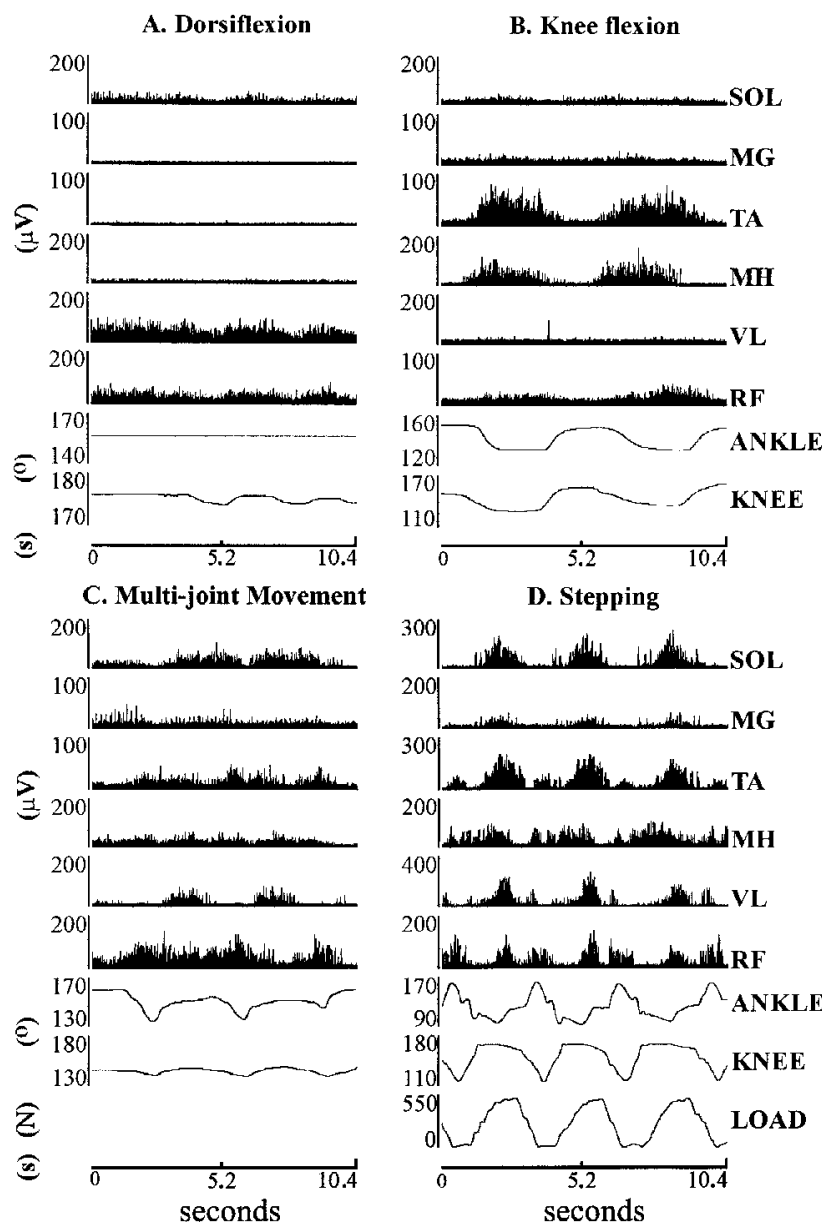

FIG. 3. Single- and multijoint movements and stepping from a clinically incomplete SCI subject (D4, reference Table 1). Electromyographic (EMG) activity $(\mu \mathrm{V})$ from the soleus (SOL) medial gastrocnemius (MG), tibialis anterior(TA), medial hamstrings $(\mathrm{MH})$, vastus lateralis (VL), and rectus femoris (RF); knee and ankle angles $\left({ }^{\circ}\right)$; and limb load in Newtons (N) during an attempted single-joint movement ( $\mathbf{A}$ and $\mathbf{B})$, multijoint movements $(\mathbf{C})$, and during weight-bearing stepping at 0.33 $\mathrm{m} / \mathrm{sec}$ with $35 \%$ body weight support (BWS) (D). The nonspecificity in the activation of the muscles was present during attempts to dorsiflex the ankle and flex the knee. Slight activation of the VL and RF occurred with attempted ankle dorsiflexion (A). When the same subject attempted to flex the knee the MH was activated, but so was the TA and RF (B). Note that the TA was not activated during attempts to dorsiflex the ankle (A). Each muscle was activated during multijoint efforts (C), although at a lower amplitude than during stepping (D). MH EMG bursts were reciprocal to the VL and RF and the TA EMG was largely synchronized with the SOL and MG during stepping with some activation during swing. 
subject D4 attempted dorsiflexion, activity occurred in the VL, RF, and SOL with no activity observable in the TA (Fig. 3A). When this subject attempted knee flexion inappropriate muscle activation was evident in the TA and RF, with the activation of the MH (Fig. 3B). During multijoint movements, clear alternating activity was only observed in the VL (Fig. 3C). During stepping, alternating activity occurred in all muscles (Fig. 3D).

In subjects $\mathrm{C} 5$ and $\mathrm{C} 6$, minimal to no EMG activity occurred during single- or multi-joint attempted movements (Fig. 4A,B). However, in both subjects (C5 and C6) during stepping alternating bursts occurred in all muscles studied (Fig. 4C) except the RF in C6.

EMG activation patterns of the clinically complete SCI subjects were similar to those observed in the incomplete SCI subjects during stepping. As expected, no muscles innervated by motoneurons below the lesion were recruited during voluntarily attempted movements in the clinically complete subjects as exemplified by subject $\mathrm{A} 8$ (Fig. 5A-E). This subject underwent 4 months of training prior to testing (Wernig and Müller, 1992). This subject repeatedly executed phases of unaided stepping with partial weight support. During some steps, assistance was needed only to place the foot at the transition from swing to stance. Small flexor bursts occurred at the initiation of the swing phase of the step cycle and extensors were ac- tive during the stance phase (Fig. 5F). As in many SCI subjects the TA activity was coactivated with plantarflexors during stance while the $\mathrm{MH}$ activity was more reciprocal with the plantarflexors, beginning in early or mid-swing and ending by mid-stance.

The degree to which the EMG patterns are attributable to a given level of training cannot be determined from the present data. However, it should be noted that when some of the clinically incomplete SCI subjects (D1, D2, D3, and D4) received Laufband therapy they improved their ability to walk overground (EU Walking; Table 2) with minimal changes in voluntary activity (CMI; Table 2 ). These data suggest that these minor changes in voluntary control of muscle activity cannot account for the marked improvements in the walking capacity these individuals achieved (also see Wernig et al., 1995, 1999).

\section{Mean EMG Amplitudes were Higher during Weight-Bearing Stepping than during Non-Weight-Bearing Voluntary Motor Tasks}

The amplitude of the EMG bursts from the six muscles of each of the 10 subjects studied was the highest during weight bearing stepping compared to non-weightbearing single- and multijoint movements in 50/60 possible conditions (six muscles and 10 subjects; Fig. 6A).
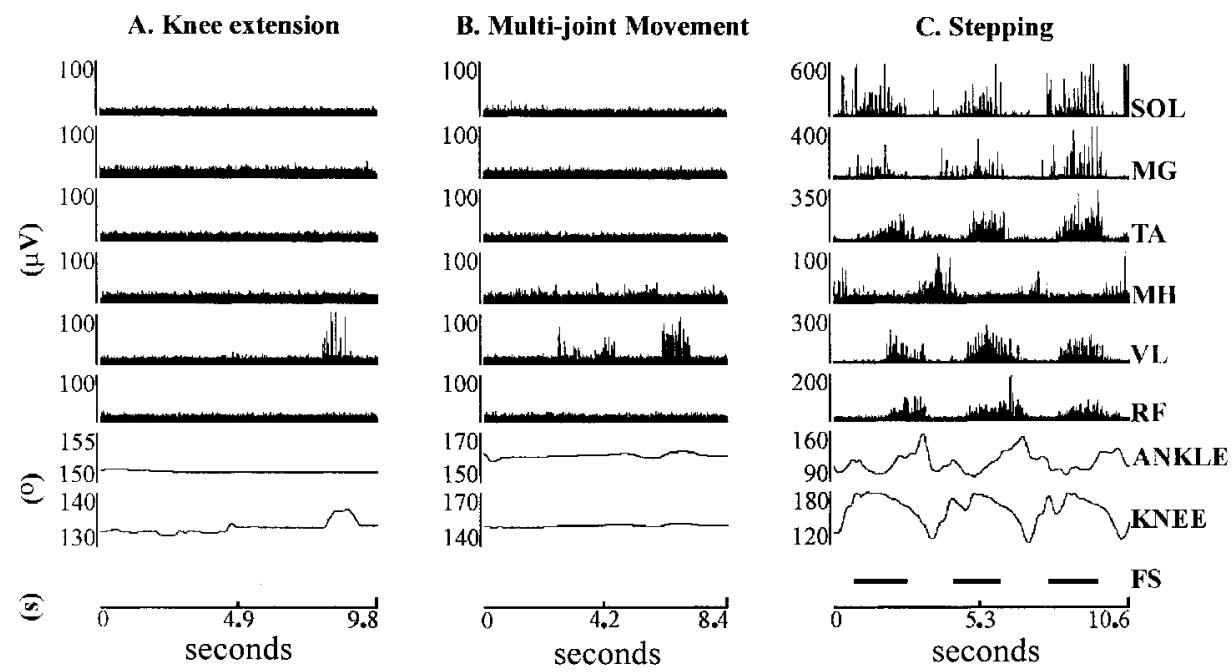

FIG. 4. Single- and multijoint movements and stepping from a clinically incomplete SCI subject (C5, reference Table 1). Electromyographic (EMG) activity $(\mu \mathrm{V})$ from the soleus (SOL), medial gastrocnemius (MG), tibialis anterior (TA), medial hamstrings $(\mathrm{MH})$, vastus lateralis (VL), and rectus femoris (RF); knee and ankle angles $\left({ }^{\circ}\right.$ ); and footswitches (black bars indicate stance phase) during an attempted single-joint movement (A), multijoint movements (B), and during weight-bearing stepping at $0.28 \mathrm{~m} / \mathrm{sec}$ with $56 \%$ body weight support (BWS) (C). Minimal EMG was observed only in the VL during attempted knee extension (A), and only the MH became more active (although no clear EMG burst) during multijoint effort (B). Minimal movement of the knee or ankle occurred. This EMG pattern contrasts with the alternating bursts in each muscle during stepping $(\mathbf{C})$. The TA was largely synchronized with the SOL and MG while the MH EMG was reciprocal to that in the VL and RF and with the ankle muscles. 

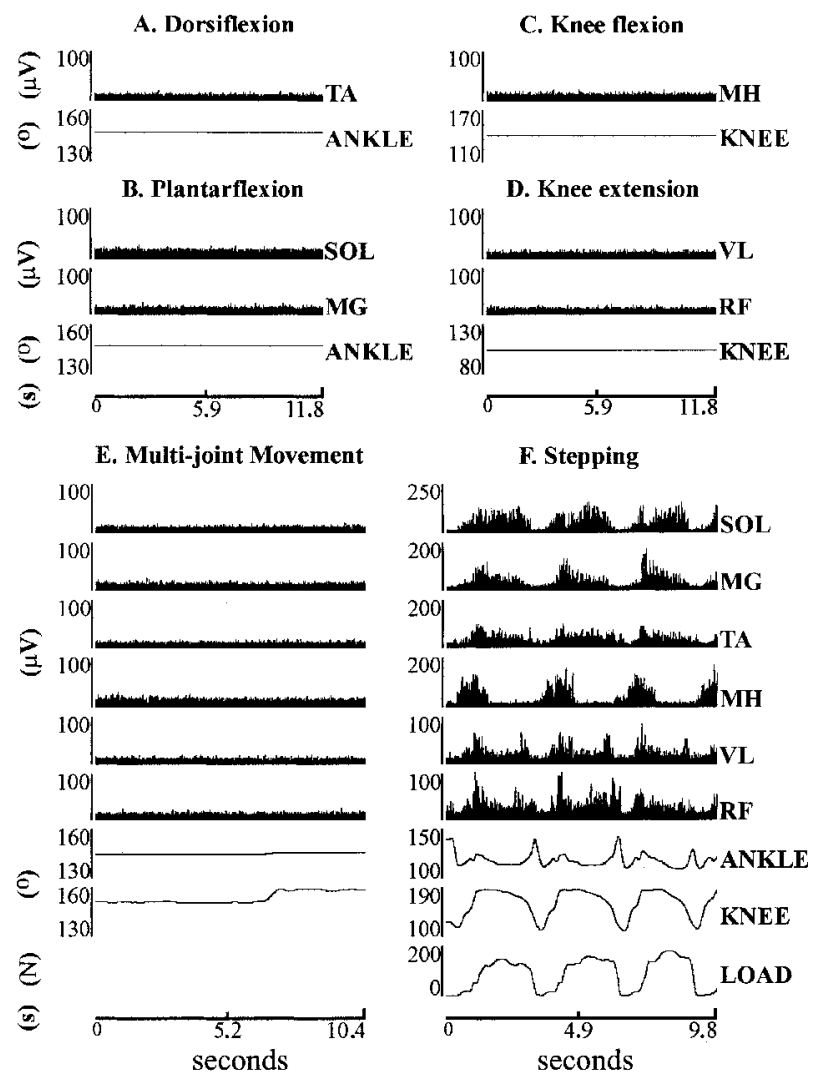

FIG. 5. Single- and multijoint movements and stepping from a clinically complete SCI subject (A8, reference Table 1). Electromyographic (EMG) activity $(\mu \mathrm{V})$ from the soleus (SOL) medial gastrocnemius (MG), tibialis anterior (TA), medial hamstrings $(\mathrm{MH})$, vastus lateralis (VL), and rectus femoris (RF); knee and ankle angles $\left(^{\circ}\right)$; and limb load in Newtons $(\mathrm{N})$ during an attempted single-joint movement (A-D), multijoint movements $(\mathbf{E})$, and during weight-bearing stepping at 0.22 $\mathrm{m} / \mathrm{sec}$ with $56 \%$ body weight support (BWS) (F). None of the muscles studied were recruited during attempted single- and multijoint movements with limbs unloaded (A-E). During stepping (F) discrete flexor bursts occurred at terminal stance/initial swing and extensors were active during stance. Similar to clinically incomplete SCI subjects, MH activity occurred during terminal swing and early stance. The TA was coactivated with plantarflexors during stance.

The EMG mean amplitudes of the SOL and the MG were higher during stepping than during non-weight-bearing voluntary motor tasks in all 10 subjects. The highest amplitudes in the TA and the VL occurred during stepping in 7/10 subjects, and in the $\mathrm{MH}$ and $\mathrm{RF}$ in 8/10 subjects. In the three subjects with no detectable EMG activity or motor score that were classified as being clinically complete, EMG activity was evident in all six muscles studied in two subjects (A8 and A10) and in three muscles (SOL, MG, and MH) in one subject (A9) during weight bearing stepping. In contrast, no detectable EMG bursts were observed during voluntary movements when the limbs were unloaded.

The amplitude of the EMG bursts in the MG and $\mathrm{MH}$ in three of the subjects with less severe SCI injuries (D1-D3) and in the TA and VL in two of these subjects (D2 and D3) during attempted multi-joint efforts was higher than those observed during single-joint efforts (Fig. 6A). Slightly higher EMG mean amplitudes during attempts to move the limbs in a step-like, multijoint action while supine were also noted in the SOL and RF of D1, whereas amplitudes in both muscles were similar or smaller in subjects D2 and D3. In subjects D4 and C1, EMG amplitudes in most cases were smaller during multijoint efforts. In the remainder of the subjects with more severe SCI injuries (A8-A10, C5 and C6), the EMG mean amplitudes were similar during single-joint and multijoint efforts (except the TA and MH of C5 and of the RF of C6). Also, in each of these five subjects (A8-A10, C5, and C6), the mean EMG amplitudes during these efforts were considerably smaller than those observed during similar efforts by the less severely impaired subjects (D1-D4).

In comparing the EMG mean amplitudes of all subjects for each muscle, a pattern similar to that observed in individual subjects was noted (Fig. 6B). The group averages of EMG mean amplitudes of the SOL, MG, MH, and VL during weight-bearing stepping were higher than during voluntary tasks without weight bearing $(p<$ $0.05)$. Although the mean TA and RF EMG mean amplitudes were significantly higher during stepping, they did not differ statistically among the motor tasks. The EMG mean amplitudes of all subjects were similar during the multijoint and single-joint tasks in all muscles studied.

\section{DISCUSSION}

This study demonstrates that the level of activation of motor pools of the lower limbs of SCI subjects during stepping exceeded that which was generated voluntarily in the absence of weight bearing. The motor pools of agonists and antagonists were more reliably and more appropriately activated during stepping compared to voluntary efforts to move single or multiple joints in similar patterns in the absence of weight bearing.

\section{Lack of Selectivity in Activation of Motor Pools during Single-Joint Efforts}

When clinically incomplete SCI subjects in the present study were asked to either flex or extend the knee or the 
ankle, agonist EMG activity was observed in muscles controlling both the ankle and knee. Multijoint flexion or extension movements of the entire limb occurred rou-

A

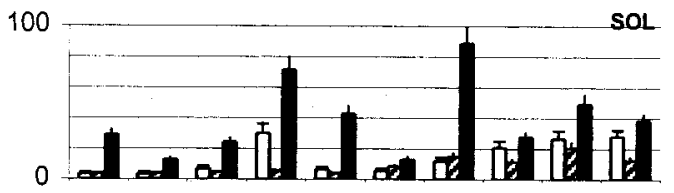

80
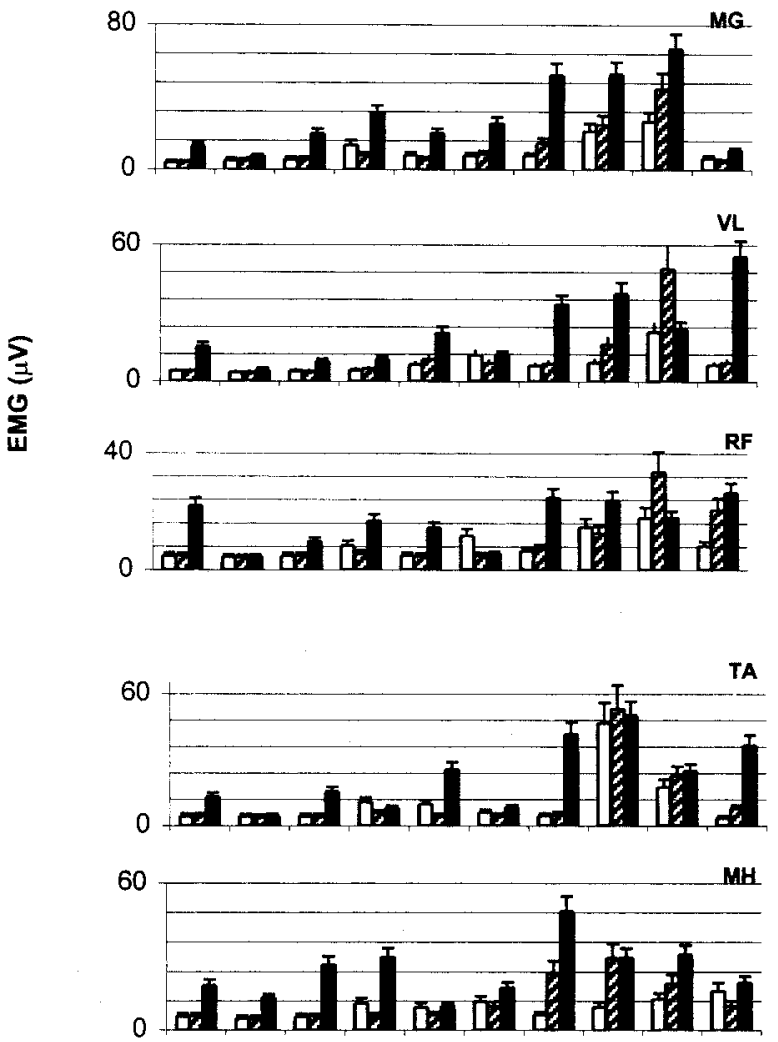

B
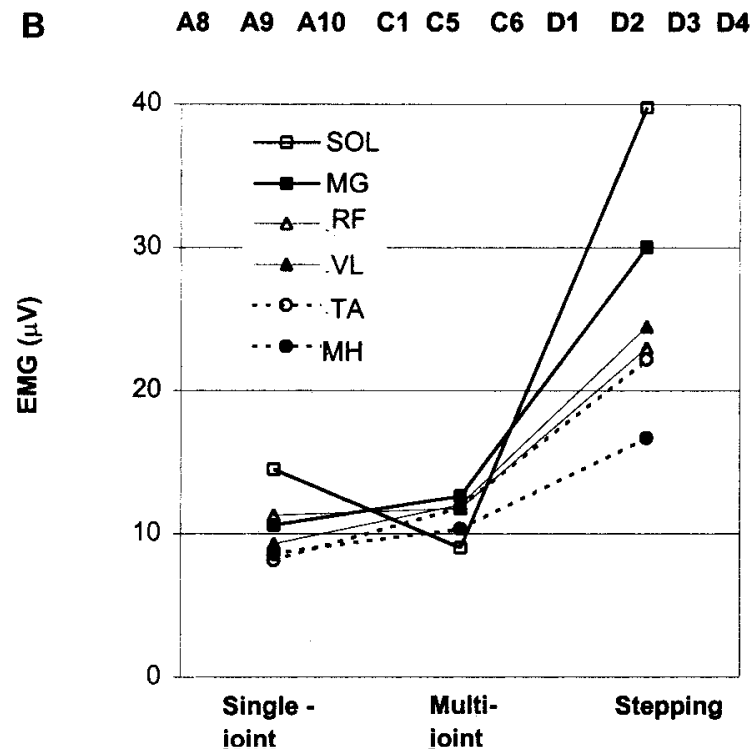

tinely when single-joint movements were attempted. Synchronous activation of agonists and antagonists were also observed during these single joint efforts. These observations are consistent with previous findings that voluntary activation of severely paralyzed limbs using Jendrassic maneuvers induces complex flexor and extensor movements involving multiple joints (Dimitrijevic et al., 1984; Wernig and Müller, 1991, 1992). The failure of clinically incomplete SCI subjects to perform single-joint movements indicates that the remaining supraspinal motor input to the spinal cord can initiate entire flexion or extension patterns, but has a limited capacity to selectively activate specific muscles of a single joint or to simultaneously inhibit the antagonist.

\section{Role of Proprioception in the Modulation of EMG Activity}

The proprioception associated with the kinematics of the moving limb and kinetics of weight bearing during stepping modulated the levels and patterns of activation of lower limb motor pools. For example, individual muscles that could not be activated during attempts to flex or extend a single-joint or during multijoint movements became active during weight-bearing stepping. In the three clinically complete subjects, that is, without any detectable voluntary activity in lower limbs, bursts of EMG activity were recorded from the majority of muscles studied during weight-bearing stepping. EMG mean amplitudes were higher during weight-bearing stepping than during non-weight-bearing voluntary motor tasks in $50 / 60$ muscles from all 10 SCI subjects studied. These

FIG. 6. Mean electromyographic (EMG) amplitudes were higher during weight-bearing stepping than during non-weightbearing voluntary motor tasks. The upper panel (A) shows average EMG mean amplitudes $(\mu \mathrm{V})$ from the soleus (SOL), medial gastrocnemius (MG), tibialis anterior (TA), medial hamstrings (MH), vastus lateralis (VL), and rectus femoris (RF) for all 10 SCI subjects studied during voluntarily attempted non-weight-bearing single-joint movements (white bars), multijoint movements approximating stepping in a supine position (black-white crossed bars), and weight-bearing stepping (black bars). Mean EMG amplitudes were the highest during weightbearing stepping in 50/60 possible conditions. The EMG mean amplitudes of all subjects combined for each muscle were highest during stepping (lower panel, B). The SOL, MG, MH, and VL had a higher group average EMG mean amplitude during weight-bearing stepping compared to conditions when the subject performed voluntary tasks without weight bearing $(p<$ 0.05). The mean TA and RF EMG amplitudes did not differ statistically among the motor tasks. The group mean EMG amplitudes during the multijoint and single-joint tasks were similar in all muscles studied. 
results strongly support that the kinematics and loading of the lower limbs provide an important source of continuous sensory signals needed for successful stepping. Some specific sensory cues (termed "rules of spinal locomotion"; Wernig et al., 1995) important for the generation of locomotion that have been identified both in animal and human studies include limb loading during stance and hip extension and shift of body weight (unloading) in terminal stance to initiate swing (Behrman and Harkema, 2000; for reviews, see Grillner, 1981; Gossard and Hultborn, 1991; Harkema, 2001).

\section{Sensory Processing by the Human Spinal Cord}

Evidence that the human spinal cord can process proprioceptive information is supported by the observations that weight supported by the lower limbs is positively correlated to the amplitude of EMG signals of the flexor and extensor muscles (Harkema et al., 1997). This relationship between loading and EMG amplitude was observed in subjects regardless of the presence or absence of injury or even the severity of the injury. These results indicate that proprioceptive input to spinal neurons can be processed so that the motor pools that generate stepping are activated in a temporally effective sequence.

In the present study, the EMG amplitudes of most of the lower limb muscles of severely injured subjects were significantly higher during weight bearing stepping than during voluntary efforts to flex or extend the knee or ankle. Furthermore, the higher EMG amplitudes during weight-bearing stepping were accompanied by the emergence of EMG bursts from other muscles that were silent during voluntary efforts to activate single or multiple motor pools without weight bearing. Both of these observations suggest that the ability of the SCI subject to voluntarily activate a muscle group in the absence of loading is not necessarily a predictable indicator of the potential utility of that muscle group to contribute to load-bearing stepping.

The comparisons of the EMG amplitude during voluntary efforts to activate a single muscle group and to activate multiple muscle groups in a step-like motion without weight support were similar. However, identifiable EMG bursts were generated from motor pools in some subjects during multijoint movements that were not present in efforts to activate a single muscle group. These data are consistent with there being some synergistic or interactive effects among multiple motor pools when attempting to generate a more complex cycling task even in the absence of loading.

It has been shown previously that the sensory modalities associated with weight-bearing stepping can facilitate activation of muscles of the lower limbs in severely incomplete SCI subjects. For example, one subject was reported to have discrete activity only from the right TA (see patient $Z$ in Wernig and Müller, 1991, 1992). However, even with this limited voluntary function this individual could walk overground for $40 \mathrm{~m}$ with a reciprocal gait using a walker without braces. This individual used a starting position that included hyperextension of one hip combined with contralateral loading, followed by ipsilateral unloading. The unloaded limb would then swing forward to begin the stance phase as the contralateral hip would extend. In this case, specific afferent signaling associated with weight-bearing stepping seems to have facilitated the recruitment of muscles during walking. In this subject, muscles that became active during stepping were not activated during voluntary attempts to generate multijoint movements without weight bearing. Similar observations in the clinically incomplete SCI subjects were made in the present study. For example, one subject (D2) after being wheelchair bound for 2 years improved from a class of 1 to 4 (EU Walking) following Laufband therapy. Her voluntary activity minimally improved (e.g., right limb 9.5 before and 10.5 after training; CMI Score; Table 2) with right hip flexors grading zero both before and after training. However, a flexion pattern including hip flexion was readily generated during an initial test of stepping using BWST and manual assistance while providing the "rules of spinal locomotion." This observation prompted the investigators to initiate the Laufband therapy on this subject. This suggests that even when individuals present low scores with voluntary manual testing the evaluation of flexion and extension patterns while stepping using BWST and manual assistance is a very important assessment when considering therapeutic approaches for the rehabilitation of walking.

\section{Interactions of Supraspinal Input and Proprioception Facilitating Stepping}

The EMG amplitudes of almost all muscles studied were higher during stepping than in single- or multijoint movements without weight bearing. It cannot be determined in these incomplete subjects whether the elevated EMG amplitudes during weight-bearing stepping were due to enhanced supraspinal control, a result of spinal facilitating events driven by proprioceptive input, or interactive spinal and supraspinal control. However, in clinically complete SCI subjects there is no dichotomy in the source of the activating input being supraspinal or spinal. In clinically complete SCI subjects, clearly the EMG mediation is initiated by the sensory input derived from the kinematics of the limb.

Previous reports have suggested that there are substantial differences between voluntary muscle activity during unloaded postures and muscle activity evoked dur- 
ing stepping on a treadmill and overground walking in incomplete SCI subjects (Wernig and Müller, 1991, 1992; Wernig et al., 1995, 1999). In the present study, we demonstrated significant muscle activity during stepping from a clinically complete SCI subject without any ability to voluntarily activate the lower limbs. This subject displayed most of the EMG activation patterns and elements of a typical step cycle, that is, stepping with flexion and extension movements with one limb independently completing a step cycle and the other needing only assistance with foot placement. However, this subject could not execute consecutive and completely unassisted bilateral step cycles on the treadmill while fully bearing weight. Comparable patterns of EMG activities to those observed here have been reported from other clinically complete SCI subjects during assisted stepping on the treadmill (Dietz et al., 1994, 1995, 1998b; Dobkin et al., 1995; Harkema et al., 1997; Stewart et al., 1991); however, complete unassisted stepping even after locomotor training has not been observed as it has in the adult complete spinal transected cat (Barbeau and Rossignol, 1987; de Leon et al., 1998a; Lovely et al., 1986).

\section{Clinical Implications}

These data provide substantial evidence that the potential recovery of walking cannot be predicted solely by the individual's ability to voluntarily generate force in a single or multiple lower limb muscle groups with the limbs unloaded. The specificity of training that improved stepping and standing in laboratory animals (de Leon et al., 1998a,b) and the SCI subjects' ability to activate motor pools at levels and patterns that approximate normal stepping may contribute to more successful recovery of overground walking. The present findings provide some of the underlying logic for why SCI subjects can improve their functional overground walking by specific locomotor training. Further, we suggest that assessments of the motor patterns of individuals where appropriate kinematics and kinetics of walking can be facilitated (on a BWST system using manual assistance) may be more reflective of locomotor potential than individual voluntary motor tests alone.

\section{ACKNOWLEDGMENTS}

This study was supported by a fund from the Deutsche Stiftung Querschnittlähmung to A. Wernig and by the following National Institute of Health grants: NS 16333, NS 38654, NRSA HD 07416 and M01 RR 00865. We would like to thank the administration and staff of the Klinikum Karlsbad-Langensteinbach, Germany, for their interest and support of this work, and the subjects who participated in the study.

\section{REFERENCES}

ANDERSSON, O., and GRILLNER, S. (1981). Peripheral control of the cat's step cycle. I. Phase dependent effects of rampmovements of the hip during "fictive locomotion." Acta Physiol. Scand. 113, 89-101.

ANDERSSON, O., and GRILLNER, S. (1983). Peripheral control of the cat's step cycle. II. Entrainment of the central pattern generators for locomotion by sinusoidal hip movements during "fictive locomotion." Acta Physiol. Scand. 118, 229239.

ANISS, A.M., GANDEVIA, S.C., and BURKE, D. (1992). Reflex responses in active muscles elicited by stimulation of low-threshold afferents from the human foot. J. Neurophysiol. 67, 1375-1384.

BARBEAU, H., and BLUNT, R. (1991). A novel interactive locomotor approach using body weight support to retrain gait in spastic paretic subjects, in: Plasticity of Motoneural Connections. A. Wernig (ed), Elsevier Science Publishers BV: Amsterdam, pps. 461-474.

BARBEAU, H., CHAU, C., and ROSSIGNOL, S. (1993). Noradrenergic agonists and locomotor training affect locomotor recovery after cord transection in adult cats. Brain Res. Bull. 30, 387-393.

BARBEAU, H., and ROSSIGNOL, S. (1987). Recovery of locomotion after chronic spinalization in the adult cat. Brain Res. 412, 84-95.

BARBEAU, H., WAINBERG, M., and FINCH, L. (1987). Description and application of a system for locomotor rehabilitation. Med. Biol. Eng. Comput. 25, 341-344.

BEHRMAN, A., and HARKEMA, S. (2000). Locomotor training after human spinal cord injury: a series of case studies. Phys. Ther. 80, 688-700.

BURKE, D., DICKSON, H.G., and SKUSE, N.F. (1991). Taskdependent changes in the responses to low-threshold cutaneous afferent volleys in the human lower limb. J. Physiol. 432, 445-458.

CAPADAY, C., and STEIN, R.B. (1986). Amplitude modulation of the soleus H-reflex in the human during standing and walking. J. Neurosci. 6, 1308-1313.

CAROLLO, J.J., PAREKH, R., and WINCHESTER, P. (1993). Calibration and force correction of the F-Scan foot pressure measurement system. RESNA 189-191.

COHEN, J. (1960). A coefficient of agreement from nominal scales. Educ. Psychol. Measure. 20, 37-46.

CONWAY, B.A., HULTBORN, H., and KIEHN, O. (1987). Proprioceptive input resets central locomotor rhythm in the spinal cat. Exp. Brain Res. 68, 643-656.

CRENNA, P., and FRIGO, C. (1987). Excitability of the soleus $\mathrm{H}$-reflex arc during walking and stepping in man. Exp. Brain Res. 66, 49-60. 
CROZIER, K.S., CHENG, L.L., GRAZIANI, V., et al. (1992). Spinal cord injury: prognosis for ambulation based on quadriceps recovery. Paraplegia 30, 762-767.

DE LEON, R.D., HODGSON, J.A., ROY, R.R., et al. (1998a). Locomotor capacity attributable to step training versus spontaneous recovery after spinalization in adult cats. J. Neurophysiol. 79, 1329-1340.

DE LEON, R.D., HODGSON, J.A., ROY, R.R., et al. (1998b). Full weight-bearing hindlimb standing following stand training in the adult spinal cat. J. Neurophysiol. 80, 83-91.

DIETZ, V., COLOMBO, G., and JENSEN, L. (1994). Locomotor activity in spinal man. Lancet 344, 1260-1263.

DIETZ, V., COLOMBO, G., JENSEN, L., et al. (1995). Locomotor capacity of spinal cord in paraplegic patients. Ann. Neurol. 37, 574-582.

DIETZ, V., CURT, A., and COLOMBO, G. (1998a). Locomotor pattern in paraplegic patients: training effects and recovery of spinal cord function. Spinal Cord 36, 380-390.

DIETZ, V., WIRZ, M., COLOMBO, G., et al. (1998b). Locomotor capacity and recovery of spinal cord function in paraplegic patients: a clinical and electrophysiological evaluation. Electroencephalogr. Clin. Neurophysiol. 109, 140-153.

DIMITRIJEVIC, M.R., FAGANEL, J., and SHERWOOD, A.M. (1984). Suprasegmentally induced motor unit in paralyzed muscles of patients with established spinal cord injury. Ann. Neurol. 16, 216-221.

DITUNNO, J.F., YOUNG, W., DONOVAN, W.H., et al. (1994). The international standards booklet for neurological and functional classification of spinal cord injury. Paraplegia 32, 70-80.

DOBKIN, B.H., HARKEMA, S.J., REQUEJO, P.S., et al. (1995). Modulation of locomotor-like EMG activity in subjects with complete and incomplete spinal cord injury. J. Neurol. Rehabil. 9, 183-190.

DUYSENS, J., and PEARSON, K.G. (1980). Inhibition of flexor burst generation by loading ankle extensor muscle in walking cats. Brain Res. 187, 321-332.

DUYSENS, J., TRIPPEL, M., HORSTMANN, G.A., et al. (1990). Gating and reversal of reflexes in ankle muscles during human walking. Exp. Brain Res. 82, 351-358.

GOSSARD, J.P. and HULTBORN, H. (1991). The organization of the spinal rhythm generation in locomotion, in: Plasticity and Motorneuronal Connections. A. Wernig (ed), Elsevier Science Publishers BV: Amsterdam, pps. 385-404.

GRILLNER, S., and ROSSIGNOL, S. (1978). On the initiation of the swing phase of locomotion in chronic spinal cats. Brain Res. 146, 269-277.

GRILLNER, S., and ZANGGER, P. (1979). On the central generation of locomotion in the low spinal cat. Exp. Brain Res. 34, 241-261.
GRILLNER, S. (1981). Control of locomotion in bipeds, tetrapods and fish, in: Handbook of Physiology: The Nervous System II. American Physiological Society: Baltimore, MD, pps. 1179-1236.

HARKEMA, S.J., HURLEY, S.L., PATEL, U.K., et al. (1997). Human lumbosacral spinal cord interprets loading during stepping. J. Neurophysiol. 77, 797-811.

HARKEMA, S.J. (2001). Neural plasticity after human spinal cord injury: application of locomotor training to the rehabilitation of walking. Neuroscientist 7, 455-468.

HUSSEY, R.W., and STAUFFER, E.S. (1973). Spinal cord injury: requirements for ambulation. Arch. Phys. Med. Rehabil. 54, 544-547.

KENDALL, H.O., KENDALL, F.P., and WADSWORTH, G.E. (1971). Muscle Testing and Function, 2nd ed. William and Wilkins: Baltimore.

LOVELY, R.G., GREGOR, R., ROY, R.R., et al. (1986). Effects of training on the recovery of full-weight-bearing stepping in the adult spinal cat. Exp. Neurol. 92, 421-435.

MAYNARD, F.M., BRACKEN, M.B., CREASEY, G., et al. (1997). International standards for neurological and functional classification of spinal cord injury. Spinal Cord 35, 266-274.

PEARSON, K.G., and DUYSENS, J. (1976). Function of segmental reflexes in the control of stepping in cockroaches and cats, in: Neuronal Control of Locomotion. R.M. Herman, S. Grillner, P. Stein, and D. Stuart (eds), Plenum Press: NY, pps. 519-537.

STEWART, J.E., BARBEAU, H., and GAUTHTER, L. (1991). Modulation of locomotor patterns and spasticity with clonidine in spinal cord injured patients. Can. J. Neurol. Sci. 18, 321-332.

WATERS, R.L., ADKINS, R.H., YAKURA, J.S., et al. (1994). Prediction of ambulatory performance based on motor scores deprived from standards of the American Spinal Injury Association. Arch. Phys. Med. Rehabil. 75, 756-760.

WATERS, R.L., YAKURA, J.S., and ADKINS, R.H. (1993). Gait performance after spinal cord injury. Clin. Orthop. 288, 87-96.

WATERS, R.L., YAKURA, J., ADKINS, R.H., et al. (1989). Determinants of gait performance following spinal cord injury. Arch. Phys. Med. Rehabil. 70, 811-818.

WERNIG, A., and MÜLLER, S. (1991). Improvement of walking in spinal cord injured persons after treadmill training, in: Plasticity of Motorneuronal Connections. Elsevier Science Publishers BV: Amsterdam, pps. 475-485.

WERNIG, A., and MÜLLER, S. (1992). Laufband locomotion with body weight support improved walking in persons with severe spinal cord injuries. Paraplegia 30, 229-238.

WERNIG, A., MÜLlER, S., NANASSY, A., et al. (1995). Laufband therapy based on "rules of spinal locomotion" is 
effective in spinal cord injured persons. Eur. J. Neurosci. 7, 823-829.

WERNIG, A., NANASSY, A., and MÜLLER, S. (1999). Laufband (treadmill) therapy in incomplete paraplegia and tetraplegia. J. Neurotrauma 16, 719-726.

WERNIG, A., NANASSY, A., and, MÜLLER, S. (2000). Laufband (LB) therapy in spinal cord lesioned persons. Prog. Brain Res. 128, 89-97.

WERNIG, A. 2000. On-line. Available. www.meb.uni-bonn.de/ wernig.

YANG, J.F., and STEIN, R.B. (1990). Phase-dependent reflex reversal in human leg muscles during walking. J. Neurophysiol. 63, 1109-1117.
Address reprint requests to: Susan J. Harkema, Ph.D. Department of Neurology A386 1000 Veteran Ave. University of California Los Angeles, Los Angeles, CA 90095-7147

E-mail: sharkema@mednet.ucla.edu

or Anton Wernig University of Bonn Wilhelmstr. 31, D-53111

Bonn, Germany

E-mail: Wernig@physio.uni-bonn.de 